\title{
Beyond Standard Model Higgs boson searches
}

\author{
Claudio Caputo* \\ Universitá degli Studi di Bari, Istituto Nazione di Fisica Nucleare \\ E-mail: claudio.caputo@cern.ch
}

\section{Paolo Francavilla}

Laboratoire de Physique Nucléaire et de Hautes Energies and Institute Lagrange de Paris,

E-mail: paolo.francavilla@cern.ch

The discovery of the Higgs boson at the Large Hadron Collider (LHC) is one of the major achievements of the ATLAS and CMS collaborations. Important questions on the nature of this new boson and on possible extensions of the scalar sector of the Standard Model have been tested by both the ATLAS and CMS collaborations with a vast variety of searches performed at a centre-of-mass energy of $7 \mathrm{TeV}$ and $8 \mathrm{TeV}$ (LHC Run 1). New searches, surpassing the Run 1 sensitivities, have been performed by the LHC collaborations with the proton proton collision data collected in 2015 at a centre-of-mass energy of $13 \mathrm{TeV}$. Two examples, the searches for $A / H \rightarrow \tau \tau$ and $H \rightarrow h h$, have been selected for a detailed description in these proceedings.

VII Workshop italiano sulla fisica pp a LHC

16-18 Maggio 2016

Pisa, Italy

\footnotetext{
* Speaker.

${ }^{\dagger}$ This work is partially supported by the ILP LABEX (under reference ANR-10-LABX-63 and ANR-11-IDEX0004-02).
} 


\section{Introduction}

One of the major achievements by the ATLAS [1] and CMS [2] collaborations during the Run1 data taking at a centre-of-mass energy of $8 \mathrm{TeV}$ at the Large Hadron Collider (LHC) is the discovery of a scalar particle, with a mass of approximately $125 \mathrm{GeV}$, and properties consistent with the Higgs boson predicted in the Standard Model (SM). An important question is whether this new particle is the SM Higgs boson or part of an extended Higgs sector. One interesting approach to answer this question is to search for additional scalars. For the first LHC collision data provided at a centre-ofmass energy of $13 \mathrm{TeV}$ and recorded by the ATLAS and CMS detectors, the collaborations have performed many searches that are motivated by a variety of models beyond the SM. The simplest extensions of the SM involve the addition of a singlet or a doublet field, known as Electroweak Singlet Models (EWS) and Two-Higgs-Doublet Models (2HDM), respectively. Many searches are motivated by these extensions and benchmarks within specific related models, such as the Minimal Supersymmetric Standard Model (MSSM). To a good approximation, the phenomenology of the Higgs sector in the MSSM is described entirely by two parameters: the mass of one of the Higgs bosons and the ratio of the vacuum expectation values, $\tan \beta$. Models in which both an additional singlet and doublet field are added in the Higgs sector have been proposed, and benchmarks in the Next to Minimal Supersymmetric Standard Model (NMSSM) have motivated several additional searches at the LHC. Finally extension with triplets fields has been searched by both the ATLAS and the CMS collaborations.

In these proceedings we present some of the searches performed with the $13 \mathrm{TeV}$ data, which are performed with an integrated luminosity of $3.2 \mathrm{fb}^{-1}$ and up to $2.3 \mathrm{fb}^{-1}$ with the ATLAS $[3,4,5,6,7]$ and CMS [8, 9, 10] detectors, respectively. In particular, we will focus on the searches of new scalar bosons in the MSSM benchmarks. Both the wide variety of searches and the precise measurement of the Higgs boson couplings performed with an integrated luminosity of $20 \mathrm{fb}^{-1}$ at $\sqrt{s}=8 \mathrm{TeV}$ by the ATLAS [11] and the CMS [12, 13, 14, 15] collaborations put stringent limits on the MSSM parameter space. In Fig. 1 is shown the Run1 results by ATLAS and CMS collaborations $[16,17]$. Among the new searches performed with the $13 \mathrm{TeV}$ data, the $A / H \rightarrow \tau \tau$ and the $H \rightarrow h h$ are particularly intriguing and already able to investigate regions of the MSSM parameter space not covered before. For this reason, these proceedings will focus on these two searches.

\section{Search for neutral Higgs bosons $H / A \rightarrow \tau \tau$}

For extensions of the Higgs sector containing two Higgs doublets, the couplings of the heavy Higgs bosons to down-type fermions can be enhanced with respect to the SM for large $\tan \beta$ values. These results in increased branching fractions to $\tau$ (and $\mu$ ) leptons and $b$ quarks, as well as a higher cross section for Higgs boson production in association with $b$-quarks. This has motivated a variety of searches in $\tau \tau, b b$ and $\mu \mu$ final states at the LHC -Run1 $[12,13,18,19,20]$ with no indication of an excess over the expected SM background.

The Run-2 search for neutral Higgs bosons decaying to $\tau$ leptons is performed in three channels by the ATLAS collaboration[3]: the $\tau_{e} \tau_{\text {had }}$ channel where one $\tau$ decays leptonically to an electron and neutrinos and the other hadronically (to hadrons and a neutrino), the $\tau_{\mu} \tau_{\text {had }}$ channel where the leptonically decaying $\tau$ produces a muon and neutrinos, and the $\tau_{\text {had }} \tau_{\text {had }}$ channel where 

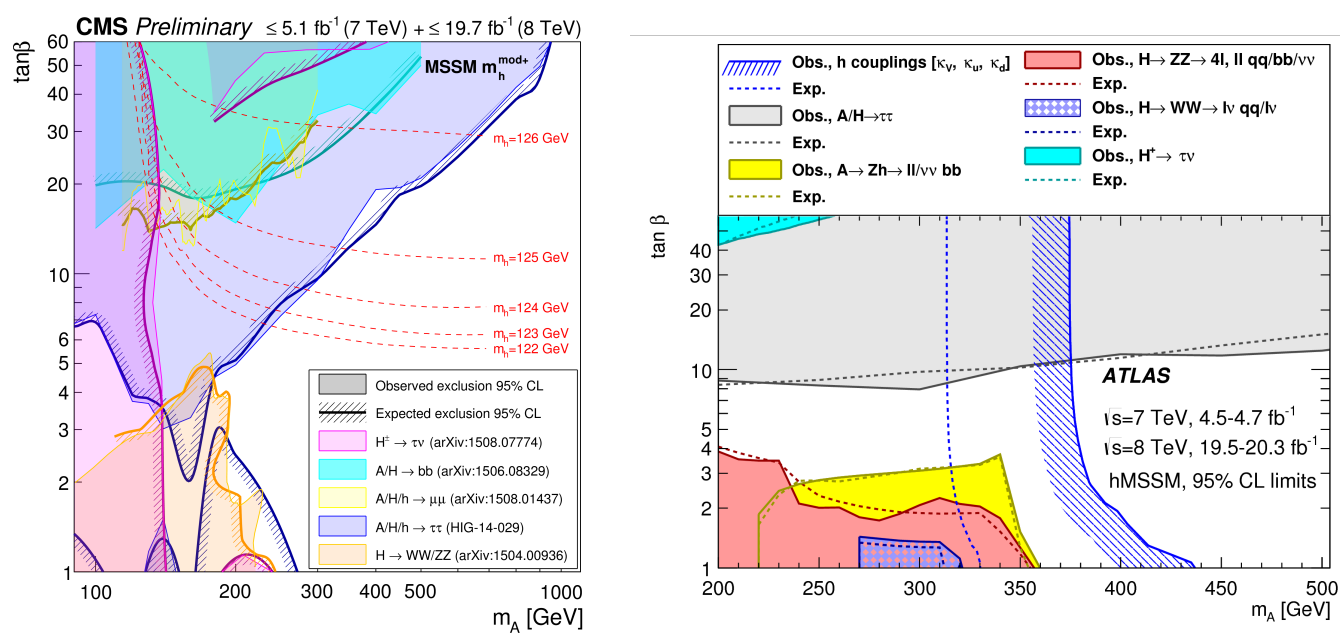

Figure 1: Run1 $m_{A}-\tan \beta$ exclusion plane by (left) CMS experiment and (right) ATLAS experiment.

both $\tau$ leptons decay hadronically. In addition to the previous three channels, the CMS collaboration extended the search in the $\tau_{e} \tau_{\mu}$ channel in which both $\tau$ 's decay leptonically [21]. In addition, the CMS collaboration divided the events into two categories based on the number of b-tagged jets: events with exactly zero b-tagged jets and events with at least one b-tagged jet.

\section{1 $\tau_{\text {had }}$ reconstruction}

The reconstruction and the identification of hadronic decays of $\tau$ leptons are critical aspects for the searches of neutral Higgs bosons $H / A$. The hadronic decays of $\tau$ leptons are predominantly characterised by the presence of one or three charged particles, accompanied by a neutrino and possibly neutral pions. In ATLAS, as reported in Ref. [22], the reconstruction of the visible decay products of the hadronic decays of $\tau$ starts with jets reconstructed from calorimeter clusters using the anti-kt algorithm with a radius of $\Delta R=0.4$ [23]. The $\tau_{\text {had }}$ candidate must have energy deposits in the calorimeters in the range $|\eta|<2.5$, with the transition region between the barrel and endcap calorimeters $(1.37<|\eta|<1.52)$ excluded, have a transverse momentum greater than $20 \mathrm{GeV}$, one or three associated tracks and an electric charge of \pm 1 . A multivariate Boosted Decision Tree (BDT) based identification is used to reject backgrounds from jets, using shower shape and track multiplicity properties. An additional dedicated likelihood-based veto is used to reduce the number of electrons misidentified as $\tau_{\text {had }}$. In the ATLAS analysis, two $\tau_{\text {had }}$ identification selections are used: "loose" and "medium" with efficiencies for true $\tau_{\text {had }}$ objects of about $65 \%$ and $55 \%$, respectively. In CMS, hadronically decaying $\tau$ leptons are reconstructed using the hadron-plusstrips algorithm [24]. The algorithm considers candidates with one charged pion and up to two neutral pions, or three charged pions, and is seeded by a jet. The neutral pions decay rapidly into two photons, and they are reconstructed as "strips" of electromagnetic particles, formed from energy depositions in the electromagnetic calorimeter ECAL. The $\tau$ decay mode is reconstructed by combining the charged hadrons with the ECAL strips. The $\tau_{\text {had }}$ candidate must have $|\eta|<2.3$ and a transverse momentum greater than $20 \mathrm{GeV}$ for the $\tau_{e} \tau_{\text {had }}$ and $\tau_{\mu} \tau_{\text {had }}$ channels, while $|\eta|<2.1$ and $p_{\mathrm{T}}>40 \mathrm{GeV}$ for the $\tau_{\text {had }} \tau_{\text {had }}$ channel. The $\tau_{\text {had }}$ candidates that are also compatible with muons or 
electrons are rejected. Jets originating from the hadronization of quarks and gluons are suppressed by requiring the $\tau_{\text {had }}$ candidate to be isolated, where the isolation variable is computed using a multivariate (MVA) approach [24].

\subsection{The $\tau_{\text {had }} \tau_{\text {had }}, \tau_{e} \tau_{\text {had }}, \tau_{\mu} \tau_{\text {had }}$ and $\tau_{e} \tau_{\mu}$ channels}

In the $\tau_{\text {had }} \tau_{\text {had }}$ channel, multi-jet events form the dominant background, and they are estimated using a data-driven techniques by both collaborations, with a fake-factor method in ATLAS, and with events in data with slightly looser isolation conditions compared to the the signal regions in CMS. These events are then weighted by the extrapolation factor from the nominal selection to this control region as measured in data events with $\tau_{\text {had }}$ candidate having the same charge. Events from other processes with a jet misidentified as a $\tau_{\text {had }}$, such as $\mathrm{W}+\mathrm{jets}$ and top-quark backgrounds, are taken from simulation and, in ATLAS, corrected using fake rates measured from data. Events with correctly identified $\tau_{\text {had }}$ are taken from simulation, with additional derived data/MC corrections.

In the $\tau_{e} \tau_{\text {had }}$ and $\tau_{\mu} \tau_{\text {had }}$ channels, the background is composed by processes involving jets misidentified as $\tau_{\text {had }}$ (multi-jet, $W+$ jets), processes with electrons misidentified as a $\tau_{\text {had }}(Z / \gamma \rightarrow$ $e e$ ), and backgrounds with a correctly identified $\tau_{\text {had }}\left(Z / \gamma \rightarrow \tau \tau\right.$ or $\left.t \bar{t} \rightarrow W^{+} W^{-} b \bar{b} \rightarrow l \tau_{\text {had }} \nu \bar{v} b \bar{b}\right)$. To suppress the $W+$ jets backgrund, a cut to reject events with transverse mass $m_{\mathrm{T}}\left(l, E_{\mathrm{T}}^{\mathrm{miss}}\right.$ ) (where $l$ is the electron or the muon, and $E_{\mathrm{T}}^{\text {miss }}$ is the missing transverse momentum) compatible with the $W$ boson are used by both the collaborations. Backgrounds from all processes that involve jets misidentified as $\tau_{\text {had }}$ are estimated simultaneously in a data-driven method. For $W+j e t s$, the measurement is performed in a region identical to the signal region, but with reversed cut on $m_{\mathrm{T}}\left(l, E_{\mathrm{T}}^{\mathrm{miss}}\right)$. The multi-jet background is measured in a region defined by inverting the isolation requirement of the electron or muon by the ATLAS collaboration, and by inverting the requirement on the relative charge of the $\tau_{\text {had }}$ with respect to the electron or to the muon by the CMS collaboration. Events with electrons misidentified as a $\tau_{\text {had }}$ are suppressed by the ATLAS collaboration with a veto of events with the mass $m\left(\tau_{\text {had }}, l\right)$ in the $\mathrm{Z}$ boson mass window, $80 \mathrm{GeV}<m\left(\tau_{\text {had }}, l\right)<110 \mathrm{GeV}$, while the CMS collaboration uses a veto of events containing a pair of opposite sign electrons or muons passing slightly looser identification and isolation conditions. Backgrounds with a correctly identified $\tau_{\text {had }}$ are taken from simulation, with additional derived data/MC corrections.

Finally, for the $\tau_{e} \tau_{\mu}$ channel studied by the CMS collaboration, the contribution from $W+$ jets is small and taken from MC. Events with same sign $(e, \mu)$ are used after subtracting the other backgrounds for the multi-jet estimate. All the other backgrounds are taken from simulation, with additional derived data/MC corrections.

The transverse invariant mass of the ditau candidate pair, $m_{\mathrm{T}}\left(\tau_{1}, \tau_{2}\right)$, is used to search for a possible signal contribution on top of the expected backgrounds by the CMS collaboration, while ATLAS used the total transverse mass, defined as $m_{\mathrm{T}}^{\text {tot }}=\sqrt{m_{\mathrm{T}}^{2}\left(\tau_{1}, \tau_{2}\right)+m_{\mathrm{T}}^{2}\left(\tau_{1}, E_{\mathrm{T}}^{\text {miss }}\right)+m_{\mathrm{T}}^{2}\left(\tau_{2}, E_{\mathrm{T}}^{\text {miss }}\right)}$. The results of both collaborations show an observed event yield compatible with the expected event yield from SM processes, within uncertainties. The $m_{\mathrm{T}}\left(\tau_{1}, \tau_{2}\right)$ and $m_{\mathrm{T}}^{\text {tot }}$ distributions for representative signal regions are shown in Fig. 2.

\subsection{Results}

The final results are provided both as limits on the cross section times branching ratio as well as a model-dependent limits on various MSSM benchmark models. The limits for all channels 

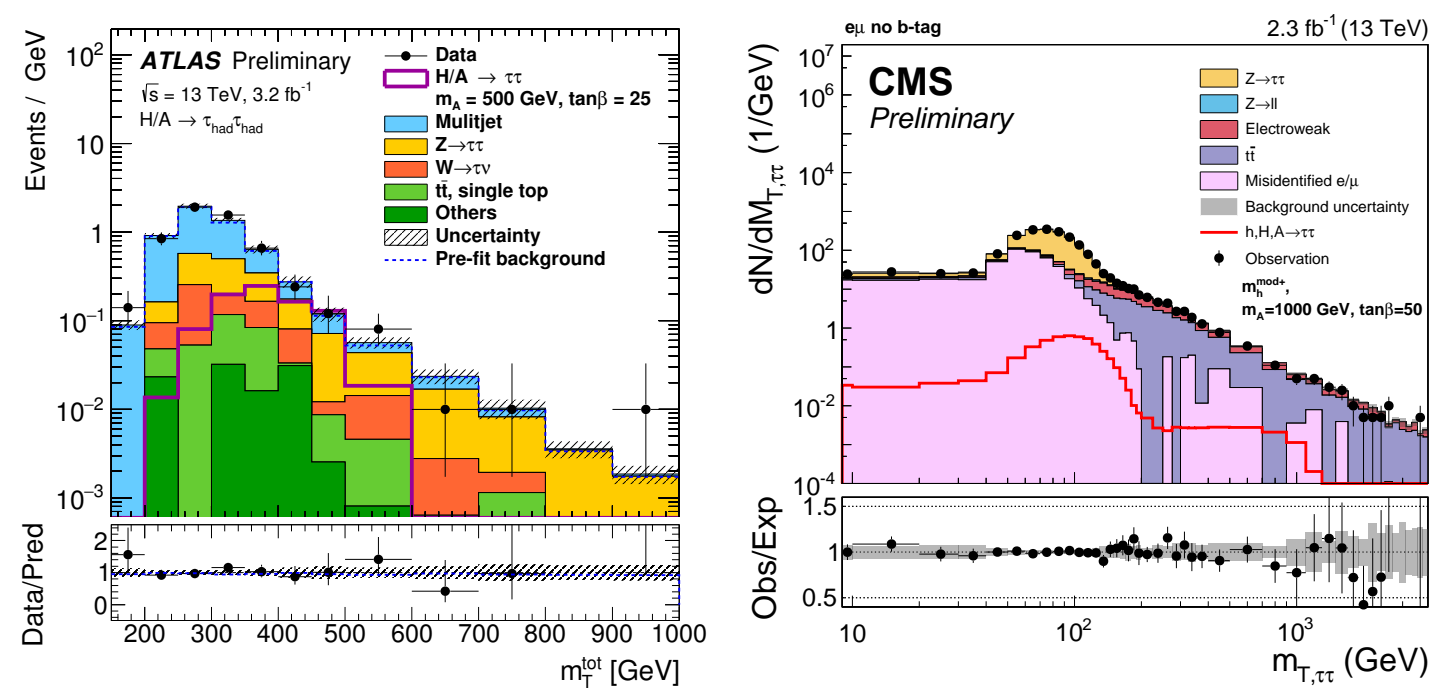

Figure 2: (Left) Post-fit plot of the total transverse mass distribution in $\mathrm{f}$ the $\tau_{\text {had }} \tau_{\text {had }}$ channel by the ATLAS collaboration. (Right) Post-fit plot of the transverse mass distribution in the no b-tag category of the $\tau_{e} \tau_{\mu}$ channel by the CMS collaboration.

combined are shown in Fig. 3 and Fig. 4 for the gluon fusion and b-associated production methods respectively, while Fig. 5 shows the expected and observed 95\% CL upper limits on $\tan \beta$ as a function of $m_{A}$ in the MSSM $m_{h}^{\bmod +}$ scenario, and the comparison with the LHC Run1 results. Already with the limited statistics recorded in 2015, the sensitivity exceeds the $8 \mathrm{TeV}$ result for $m_{H}>750 \mathrm{GeV}$ for ATLAS and $m_{H}>350 \mathrm{GeV}$ for CMS.
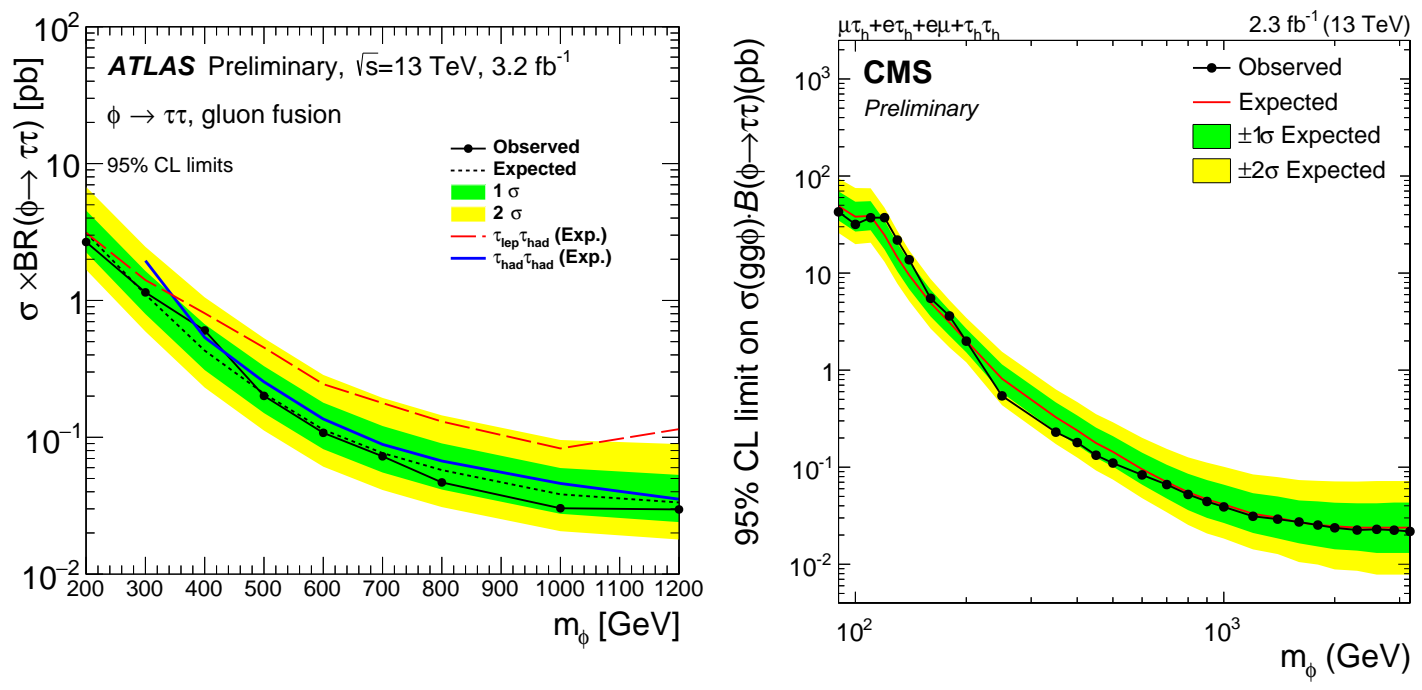

Figure 3: Observed and expected 95\% CL upper limits on the product of cross section and the branching fraction $\sigma(p p \rightarrow \phi) \times B(\phi \rightarrow \tau \tau)$ obtained by the ATLAS collaboration (left) and by the CMS collaboration (right) for the gluon-gluon fusion production. 

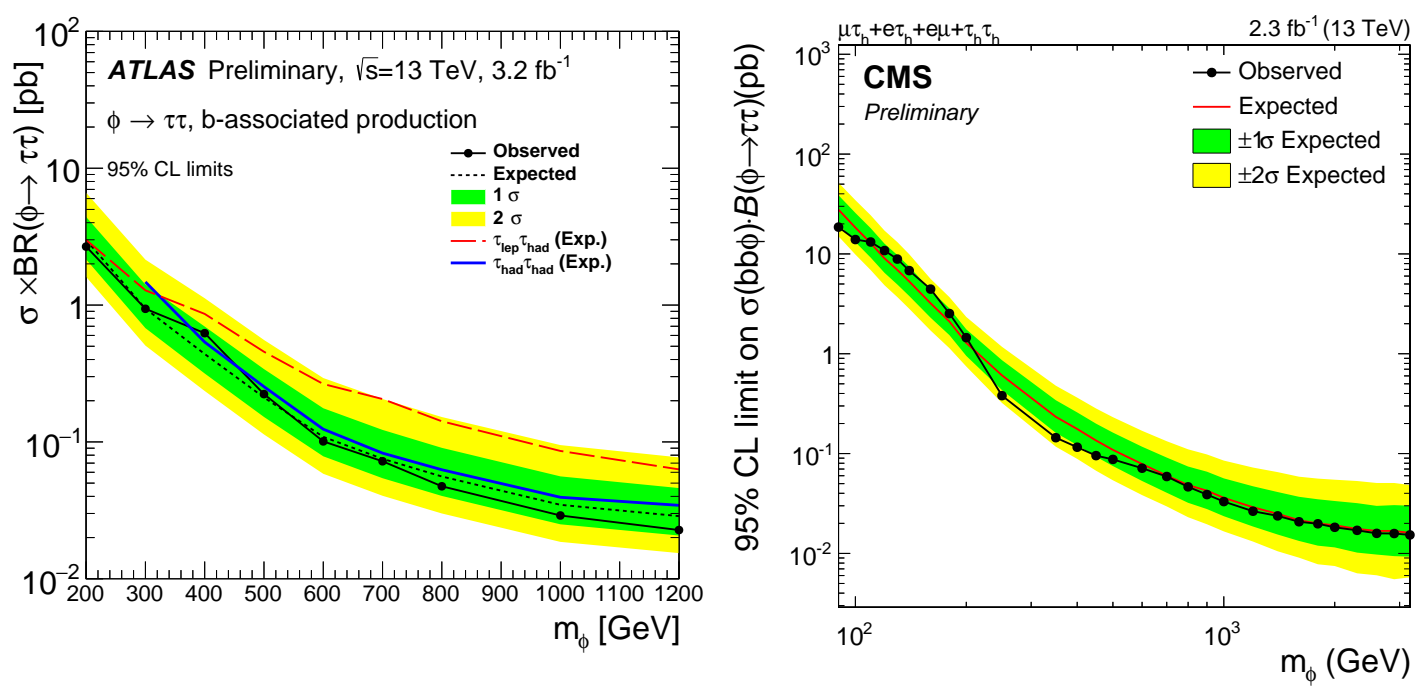

Figure 4: Observed and expected 95\% CL upper limits on the product of cross section and the branching fraction $\sigma(p p \rightarrow \phi) \times B(\phi \rightarrow \tau \tau)$ obtained by the ATLAS collaboration (left) and by the CMS collaboration (right) for the $b$-associated production.
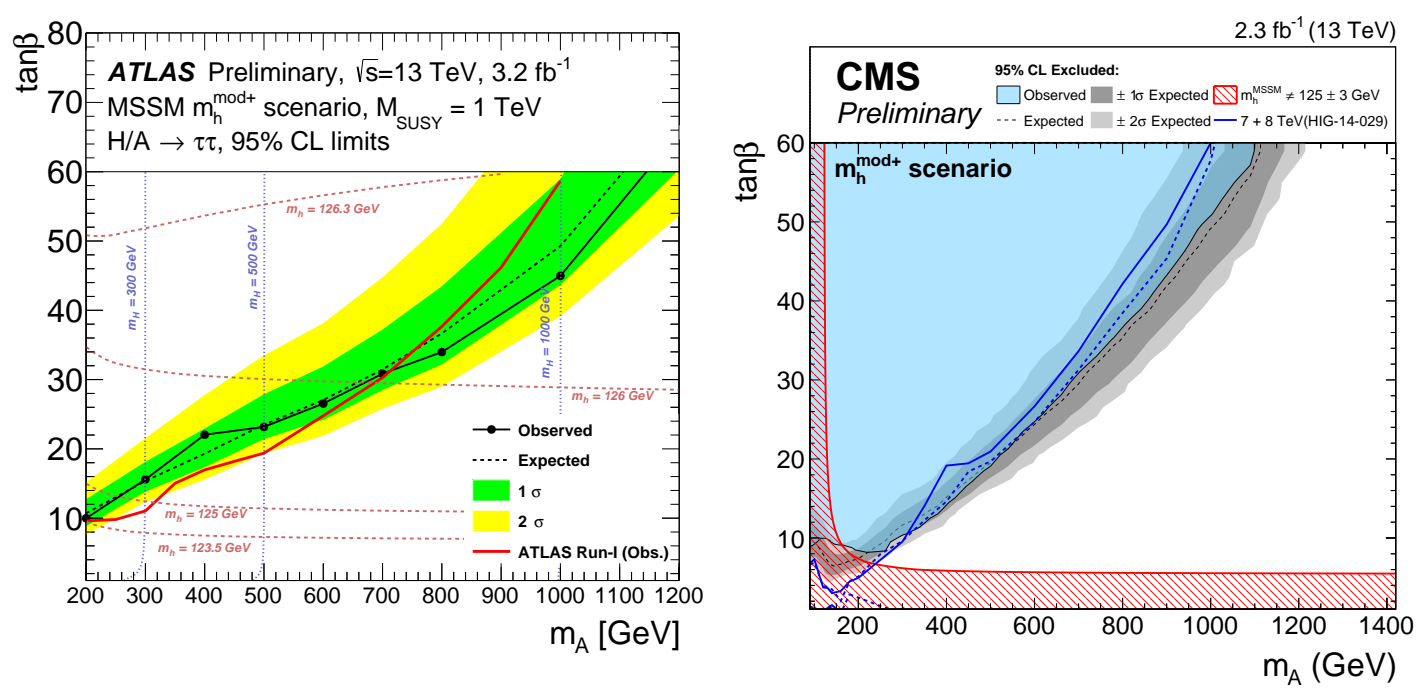

Figure 5: The expected and observed 95\% CL upper limits on $\tan \beta$ as a function of $m_{A}$ in the MSSM $m_{h}^{\text {mod }+}$ scenario for ATLAS (left) and CMS (right). 


\section{Search for Higgs boson pair production}

The Higgs boson pair production could be used to investigate both the SM and the BSM scenarios.

Searches for non-resonant $h$ production give information on the trilinear coupling present in the SM Higgs potential fields; the same searches could spot out discrepancies with respect to the SM $\lambda_{\text {hhh }}$ introduced by anomalous couplings and new physics.

Similar searches in the resonant regime could be used to explore the BSM world; new particles, introduced by extensions in the $\mathscr{L}_{S M}$, could decay in a couple of Higgs SM like boson (Di-Higgs). The experience acquired during the $8 \mathrm{TeV}$ data taking period at LHC for SM Higgs search is used for seek different final state configuration of the Di-Higgs.

In this paragraph an overview of the latest resonant results, provided by ATLAS and CMS experiment for $13 \mathrm{TeV}$ collision, will be presented. Both collaborations have produced results with $8 \mathrm{TeV}$ data [25][26] in many Di-Higgs decay configurations as shown in Fig. 6.
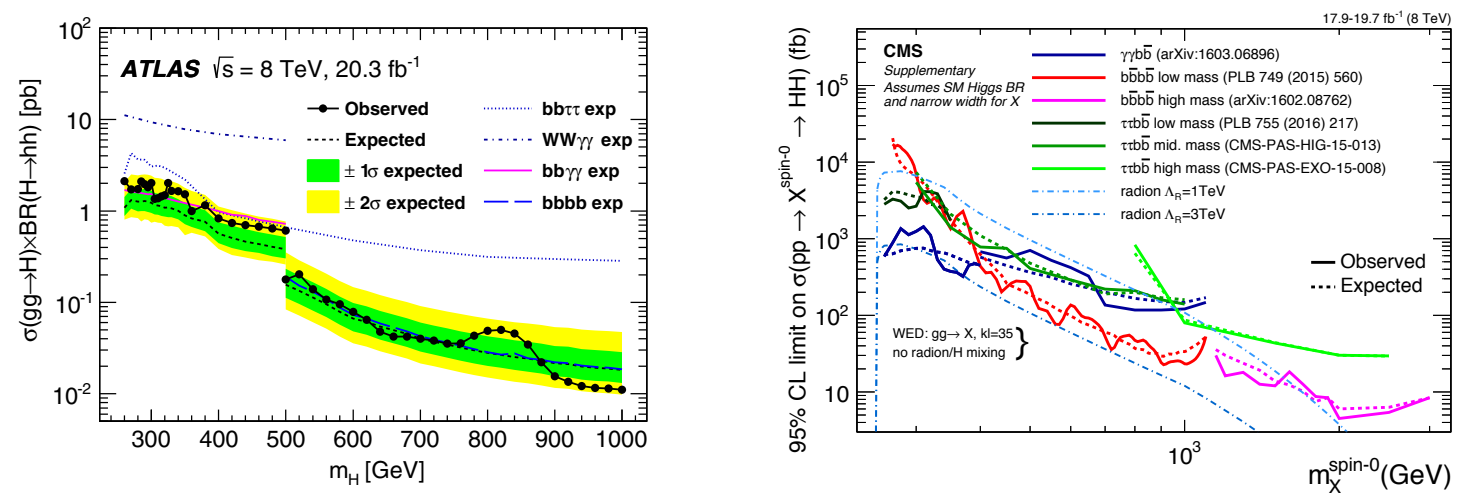

Figure 6: (left) ATLAS combination Higgs boson pair production in the $h h \rightarrow b b \tau \tau, \gamma \gamma W W^{*}, \gamma \gamma b b, b b b b$ channels with $8 \mathrm{TeV}$ data, (right) CMS observed and expected 95\% CL upper limits on the product of cross section and the branching fraction $\sigma(g g \rightarrow X) \times B(X \rightarrow h h)$ obtained by different analyses assuming spin- 0 hypothesis.

\section{1 $H \rightarrow h h \rightarrow b b b b$ channel}

The Higgs boson decay mode in $\mathrm{b}$ quarks has the highest branching ratio, this imply that a Di-Higgs final state, with each Higgs dacaying into a b pair, it's the most prominent one between the others. Although search of this kind of final state should take into account the overwhelming multi-jet background that could limit the sensitivity of the channel with respect to other finale states.

ATLAS and CMS collaborations analysis scan a mass range $260 \mathrm{GeV}<m_{H}<3000 \mathrm{GeV}$, particularly CMS cover from $260 \mathrm{GeV}$ to $1200 \mathrm{GeV}$ and ATLAS from $500 \mathrm{GeV}$ to $3000 \mathrm{GeV}$. Both analysis have different strategy for different $m_{H}$ ranges: low-mass region $\left(260 \mathrm{GeV}<m_{H}<400\right.$ $\mathrm{GeV}$ ), medium-mass ragion $\left(400 \mathrm{GeV}<m_{H}<1200 \mathrm{GeV}\right)$, boosted region $\left(1200 \mathrm{GeV}<m_{H}<\right.$ $3000 \mathrm{GeV}$ ). The categorisation is chosen to maximise to significance of the analysis.

The results are shown in Fig. 7. 

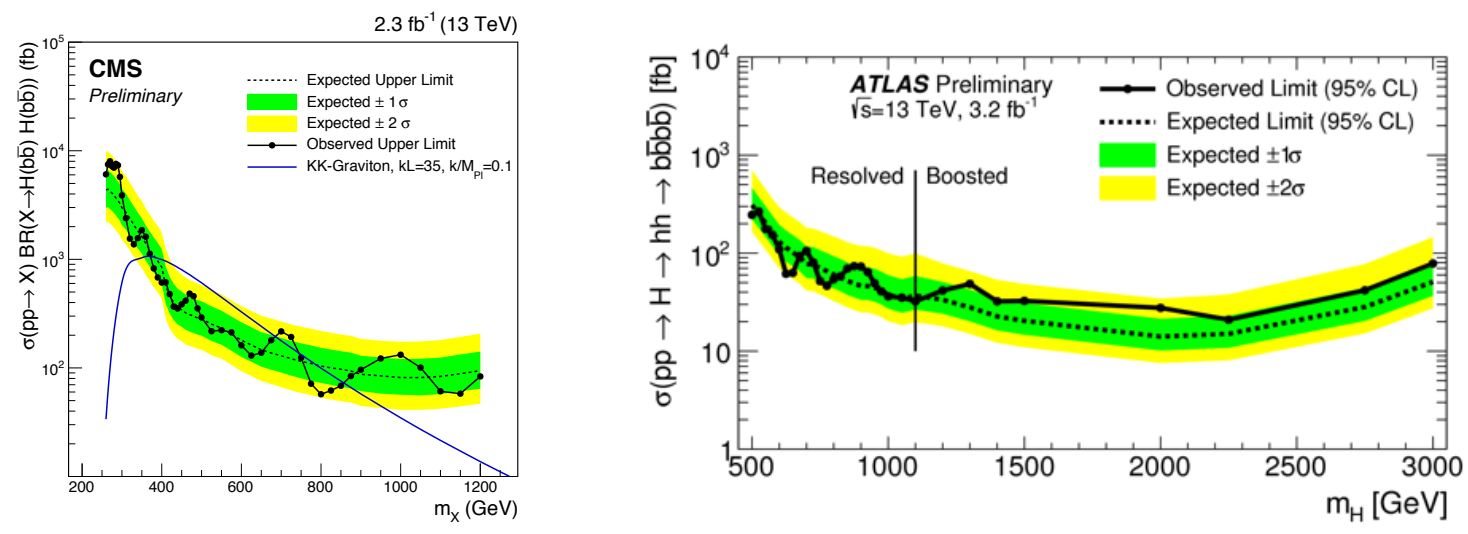

Figure 7: (left) CMS: The observed and expected upper limits on the cross section for a spin-2 resonance $H \rightarrow h h \rightarrow b b b b$ at a $95 \%$ confidence level using data corresponding to an integrated luminosity of $2.3 \mathrm{fb}^{-1}$ at $13 \mathrm{TeV}$ using the asymptotic $C L_{S}$ method, (right) ATLAS: The expected and observed upper limit for $p p \rightarrow H \rightarrow h h \rightarrow b b b b$ with fixed $\Gamma_{H}=1 \mathrm{GeV}$, at the $95 \%$ confidence level .

\section{2 $H \rightarrow h h \rightarrow b b \gamma \gamma$ channel}

The $b b \gamma \gamma$ final state is particularly promising for the DiHiggs search, as it benefits from the large branching fraction of the $h \rightarrow b b$ decay and the clean di-photon signal, due to high $m_{\gamma \gamma}$ resolution, on top of a smooth continuum di-photon background from multi-jet and multi-photon SM processes.

The analysis performed by ATLAS collaboration scans masses in the range $275 \mathrm{GeV}<m_{H}<$ $400 \mathrm{GeV}$. A counting approach is adopted in order to estimate the number of signal and background events.

Observed and expected 95\% CL upper limits are shown in Fig. 8.

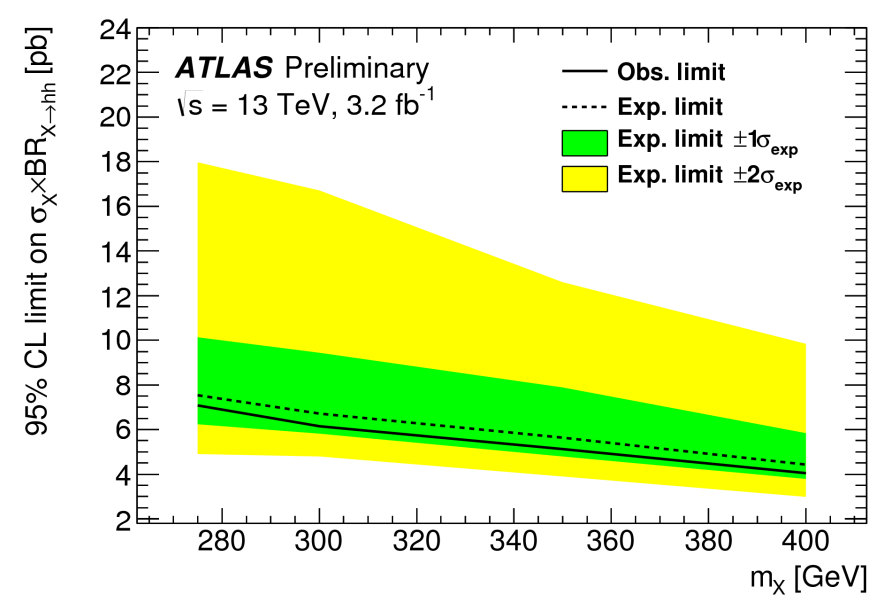

Figure 8: Observed and expected 95\% CL upper limits on $\sigma(p p \rightarrow H) \times B R(H \rightarrow h h)$ 


\section{3 $H \rightarrow h h \rightarrow b b \tau \tau$ channel}

The $b b \tau \tau$ channel can exploit the presence of the $\tau$ leptons to suppress the multi-jet background.

The analysis performed by CMS collaboration scans a $260 \mathrm{GeV}<m_{H}<900 \mathrm{GeV}$ and combine three different $\tau \tau$ final state: $\mu \tau_{h}, e \tau_{h}$ and $\tau_{h} \tau_{h}$, where $\tau_{h}$ stands for the hadronic decays of a $\tau$. The finale $m_{H}$ shape is constructed using a dedicated kinematic fit procedure.

Observed and expected 95\% CL upper limits are shown in Fig. 9.

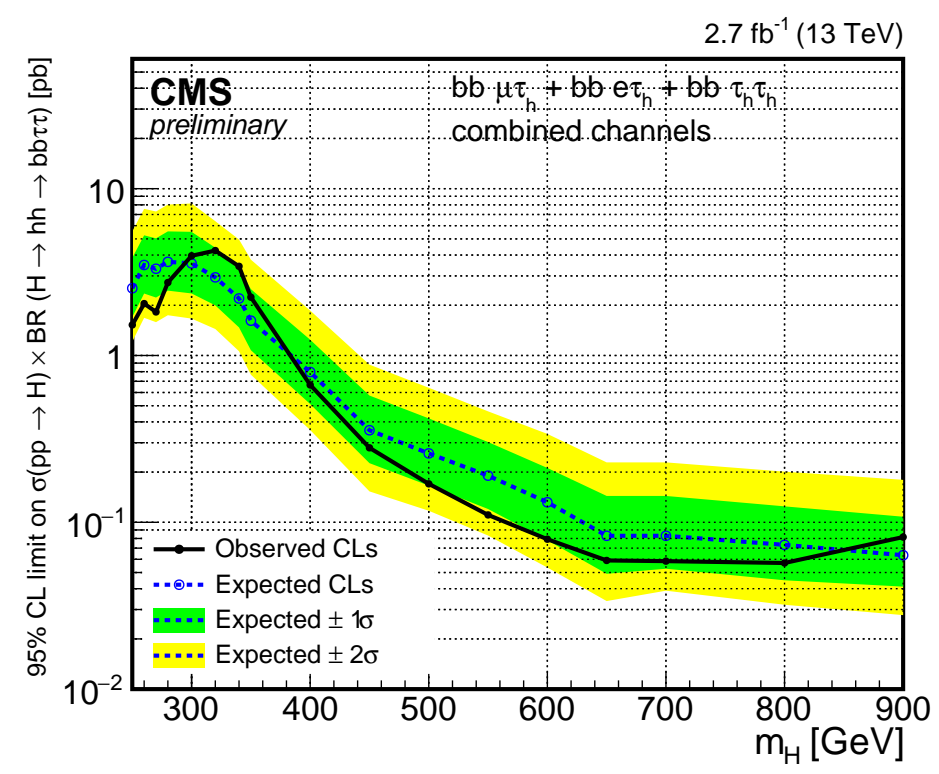

Figure 9: Observed and expected 95\% CL upper limits on $\sigma(p p \rightarrow H) \times B R(H \rightarrow h h \rightarrow b b \tau \tau)$ from the combination of the three channels as a function of the mass of the resonance $m_{H}$

\section{4 $H \rightarrow h h \rightarrow b b W W$ channel}

The search for resonant Higgs pair production, $H \rightarrow h h$, where one of the h decays as $h \rightarrow b b$, and the other as $H \rightarrow W W \rightarrow l v l v$ (where 1 is either an electron or a muon) is performed by CMS collaboration using LHC proton-proton collision data at $13 \mathrm{TeV}$. The analysis focuses on the invariant mass distribution of the b-jet pair, searching for a resonant-like excess compatible with the $\mathrm{h}$ boson mass in combination with a boosted decision tree discriminant based on kinematic information. The dominant background is tt production with smaller contributions from Drell-Yan and single top processes. Fig. 10 shows the result obtained.

\subsection{Results}

Results for Di-Higgs resonant searches at $13 \mathrm{TeV}$ don't show presence of a new resonance decaying in two SM Higgs bosons. Exclusion limits are set for all the different final states. 


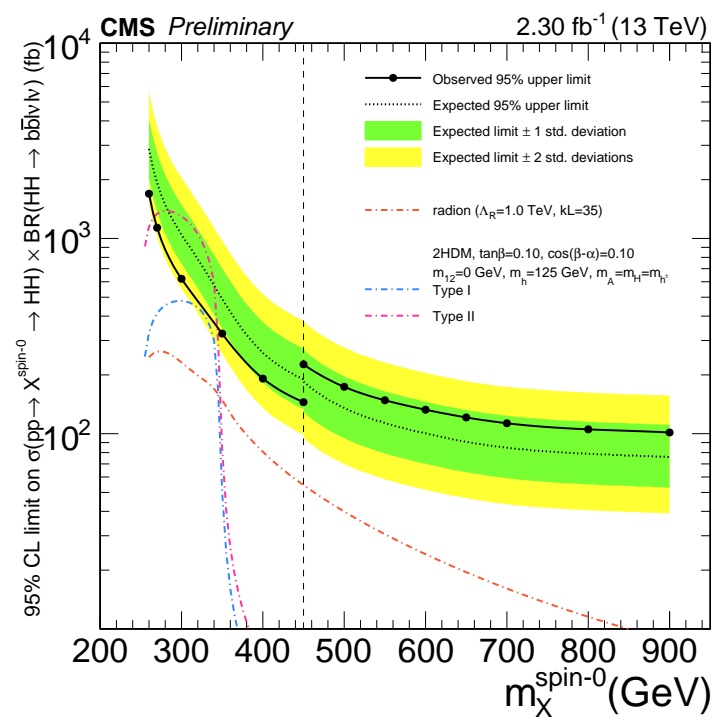

Figure 10: Observed and expected 95\% CL upper limits on $\sigma(p p \rightarrow H) \times B R(H \rightarrow h h \rightarrow b b l v l v)$

\section{Conclusions}

The discovery of a scalar boson at the LHC strengthened the interest for searches of extension of the scalar sector of the Standard Model. A vast variety of searches have been performed by the ATLAS and CMS collaboration, using the data collected during the Run 1. Thanks to protonproton collisions at a center of mass energy of $13 \mathrm{TeV}$, the data collected in 2015 allowed the LHC collaborations to extend the reach in sensitivity in the searches for Higgs bosons beyond the SM. Searches for additional Higgs bosons performed so far have shown consistency with the SM predictions, although the data that will be collected in the next few years can enlighten the nature of the Higgs sector.

\section{References}

[1] G. Aad et al., "The ATLAS Experiment at the CERN Large Hadron Collider," JINST, vol. 3, p. S08003, 2008.

[2] S. Chatrchyan et al., "The CMS experiment at the CERN LHC," JINST, vol. 3, p. S08004, 2008.

[3] "Search for Neutral Minimal Supersymmetric Standard Model Higgs Bosons $H / A \rightarrow \tau \tau$ produced in $p p$ collisions at $\sqrt{s}=13 \mathrm{TeV}$ with the ATLAS Detector," Tech. Rep. ATLAS-CONF-2015-061, CERN, Geneva, Dec 2015.

[4] M. Aaboud et al., "Search for pair production of Higgs bosons in the $b \bar{b} b \bar{b}$ final state using proton-proton collisions at $\sqrt{s}=13 \mathrm{TeV}$ with the ATLAS detector," 2016.

[5] "Search for Higgs boson pair production in the $b \bar{b} \gamma \gamma$ final state using pp collision data at $\sqrt{s}=13 \mathrm{TeV}$ with the ATLAS detector," Tech. Rep. ATLAS-CONF-2016-004, CERN, Geneva, Mar 2016.

[6] M. Aaboud et al., "Search for charged Higgs bosons produced in association with a top quark and decaying via $H^{ \pm} \rightarrow \tau v$ using $p p$ collision data recorded at $\sqrt{s}=13 \mathrm{TeV}$ by the ATLAS detector," Phys. Lett., vol. B759, pp. 555-574, 2016. 
[7] M. Aaboud et al., "Search for charged Higgs bosons produced in association with a top quark and decaying via $H^{ \pm} \rightarrow \tau v$ using $p p$ collision data recorded at $\sqrt{s}=13 \mathrm{TeV}$ by the ATLAS detector," Phys. Lett., vol. B759, pp. 555-574, 2016.

[8] "Search for resonant Higgs boson pair production in the $b \bar{b} \tau^{+} \tau^{-}$final state," Tech. Rep. CMS-PAS-HIG-16-013, CERN, Geneva, 2016.

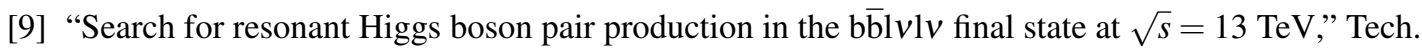
Rep. CMS-PAS-HIG-16-011, CERN, Geneva, 2016.

[10] "Search for resonant pair production of Higgs bosons decaying to two bottom quark-antiquark pairs in proton-proton collisions at $13 \mathrm{TeV}$," Tech. Rep. CMS-PAS-HIG-16-002, CERN, Geneva, 2016.

[11] G. Aad et al., "Search for new phenomena in events with at least three photons collected in $p p$ collisions at $\sqrt{s}=8 \mathrm{TeV}$ with the ATLAS detector," Eur. Phys. J., vol. C76, no. 4, p. 210, 2016.

[12] V. Khachatryan and etal, "Search for neutral mssm higgs bosons decaying to $\mu^{+} \mu$ in pp collisions at," Physics Letters B, vol. 752, pp. 221 - 246, 2016.

[13] V. Khachatryan and etal, "Search for neutral mssm higgs bosons decaying into a pair of bottom quarks," Journal of High Energy Physics, vol. 2015, no. 11, pp. 1-43, 2015.

[14] V. Khachatryan and etal, "Search for a charged higgs boson in pp collisions at $\sqrt{s}=8$ tev," Journal of High Energy Physics, vol. 2015, no. 11, pp. 1-64, 2015.

[15] "Search for a doubly-charged Higgs boson with $\sqrt{s}=8 \mathrm{TeV} p p$ collisions at the CMS experiment," Tech. Rep. CMS-PAS-HIG-14-039, CERN, Geneva, 2016.

[16] G. Aad and etal, "Constraints on new phenomena via higgs boson couplings and invisible decays with the atlas detector," Journal of High Energy Physics, vol. 2015, no. 11, pp. 1-52, 2015.

[17] "Summary results of high mass BSM Higgs searches using CMS run-I data," Tech. Rep. CMS-PAS-HIG-16-007, CERN, Geneva, 2016.

[18] G. Aad et al., "Search for neutral Higgs bosons of the minimal supersymmetric standard model in pp collisions at $\sqrt{s}=8 \mathrm{TeV}$ with the ATLAS detector," JHEP, vol. 11, p. 056, 2014.

[19] G. Aad et al., "Search for the neutral Higgs bosons of the Minimal Supersymmetric Standard Model in $p p$ collisions at $\sqrt{s}=7 \mathrm{TeV}$ with the ATLAS detector," JHEP, vol. 02, p. 095, 2013.

[20] G. Aad et al., "Search for neutral MSSM Higgs bosons decaying to $\tau^{+} \tau^{-}$pairs in proton-proton collisions at $\sqrt{s}=7 \mathrm{TeV}$ with the ATLAS detector," Phys. Lett., vol. B705, pp. 174-192, 2011.

[21] C. Collaboration, "Search for a neutral MSSM Higgs boson decaying into $\tau \tau$ at $13 \mathrm{TeV}$," 2016.

[22] ATLAS Collaboration, "Reconstruction, Energy Calibration, and Identification of Hadronically Decaying Tau Leptons in the ATLAS Experiment for Run-2 of the LHC," ATL-PHYS-PUB-2015-045, Nov 2015.

[23] M. Cacciari, G. P. Salam, and G. Soyez, "The anti-kt jet clustering algorithm," JHEP, vol. 04, p. 063, 2008.

[24] V. Khachatryan et al., "Reconstruction and identification of ÏǏ lepton decays to hadrons and $\hat{\mathrm{I}}_{i}$ at CMS," JINST, vol. 11, no. 01, p. P01019, 2016.

[25] A. Collaboration, "Searches for higgs boson pair production in the $h h \rightarrow b b \tau \tau, \gamma \gamma w W^{*}, \gamma \gamma b b, b b b b$ channels with the atlas detector," Phys. Rev. D, vol. 92, p. 092004, Nov 2015.

[26] "Higgs pag summary plots," https://twiki.cern.ch/twiki/bin/view/CMSPublic/SummaryResultsHIG. 